\section{Comparison between conventional and recent culture methods for detection of fish mycobacteria}

\author{
Mahmoud E. Hatem ${ }^{1}$, Essam A. Nasr', Mohamed S. \\ Marzouk ${ }^{1}$, Fatma A. S. Mohamed ${ }^{3}$, Mahmoud $H$. \\ Mohmed $^{4}$
}

${ }^{1}$ Department of microbiology, Faculty of Veterinary Med., Cairo University.

${ }^{2}$ Department of microbiology, Serum and Vaccine Research Institute

3 Department of microbiology, National Institute of Oceanography and Fisheries.

${ }^{4}$ Department of fish diseases, faculty of veterinary medicine, New Valley University.
* Corresponding Author

Mahmoud H. Mohmed, Department of fish

diseases, Faculty of Veterinary Medicine, New Valley University, New Valley 72511,

Egypt.Dm4467201@vet.nvu.edu.eg

Submitted $5 / 1 / 2021$

Accepted 6/1/2021

\begin{abstract}
The present study aimed to isolate and identify mycobacteria from Nile tilapia and fresh water as well as ornamental fish and aquarium water in Sohag Assuit and Qena Governorates. Conventional and recent methods (MB-Redox system) were used for isolation and detection of mycobacteria with comparison between them regarding to the rate of isolation time for growth and growth character of colonies. Three isolates were isolated from fresh water and identified as unclassified atypical mycobacteria and four isolates were recovered from aquarium water and identified as $M$. fortuitum and M. marinum. Eleven isolates were isolated from goldfish and identified as M. marinum, M. fortuitum and unclassified atypical mycobacteria. The recovery rate of MB-Redox system for mycobacteria was $12 \%$, while in case of L-J medium was $10.66 \%$. The mean time for detection of M. marinum was 3-7 days and 7-10 days for MBRedox system and Lowenstein - Jensen medium, respectively. The mean time for detection of M. fortuitum was 1-3 days for MB-Redox system, while in case of L-J medium was 3-6 days. The mean time for detection of other unidentified atypical mycobacteria was 3-8 days for MB-Redox system, while in case of L-J medium was 3-14 days. The detection of mycobacterial growth on MB-Redox system at different times revealed that the color of MB- Redox tubes appear yellow at time of inoculation changed to pink color after 3 days. The colonies appear as red particles on the surface. After 15 days, the color of MB-Redox medium changed to yellow with red particles on the surface.
\end{abstract}

Keywords: MB-Redox, mycobacteriosis, ornamental fish, tilapia fish

\section{Introduction}

Fish mycobacteriosis once known as piscine tuberculosis, is a chronic progressive disease caused by several species of the genus Mycobacterium. Mycobacterial species are capable of causing serious and costly disease in most vertebrates including humans, livestock and fish. While commonly reported in aquaculture and the fish aquaria trade, reports in wild fish have been infrequent (Jacobs et al., 2009). The high susceptibility of some ichthyic species toward mycobacteria makes them perfect carriers, able to transmit pathogens to other fish and human. In recent years, several studies have reported the presence of mycobacteria in ornamental as well $\mathrm{s}$ farmed species (Macri et al., 2008). A variety of different media for the cultivation of mycobacteria have been described but a few of them are in use today (Satio, 1998). Those currently used can be characterized by three basic types. The first is eggbased media represented by Ogawa and LowensteinJensen medium. The second type is agar-based media, the most common one is Middlebrook $7 \mathrm{H} 10$ and 7H11. The third type is liquid media such as Middle 
brook 7H9. Several weeks of incubation may be required for the isolation of mycobacteria on solid media. Substantial improvement in the time to detection and recovery rate was realized by using broth-based culture system such as the BACTEC 460 TB, Septi-Chek AFB, BACTEC 9000 and MGIT. A novel system is MB-Redox which is a modified serum-supplemented Kirchner medium containing iodonitro-tetrazolium salt as indicator of microbial growth (Satio, 1998).

\section{Materials and Methods}

\section{Samples:}

A. A total number of 240 Tilapia fish were collected from Nile River (at Sohag 85 fish, at Assuit 80 fish and at Qena 75 fish). Sampled fish were chosen either with necrotic skin lesions or with ascites. Tissue samples from skin, skeletal muscles, gills, kidney, spleen and liver were taken for bacteriological examination.

B. Sixty fantail gold fishes (Carassius auratus) were collected from pet shops. Sampled fish were showed ulcers on the head or body, exophthalmia and ascites in some of them. Samples were aseptically collected from lesions using the needle of a platinum loop. Tissue samples were taken from ulcers, kidney, spleen, liver, eye, gills and ascitic fluid for bacteriological examination. A direct film was prepared as an impression smear from each lesion as well as from ascetic fluid.

C. Moreover, 50 water samples were collected in sterile glass bottles from different locations of Nile River in Sohag, Assuit and Qena Governorates.

D Forty water samples from aquaria were collected in sterile glass bottles.

\section{Isolation of mycobacteria:}

I- Conventional culture methods [Lowenstein - Jensen medium (Difco)] (Marks, 1972):

-Organs and tissue samples showing gross lesions or congestion were cut and divided into small parts in a small sterile mortar containing washed sterilized sand.

-The tissue was homogenized in sterilized distilled water, then the homogenate was transferred into a sterilized centrifuge tube of 30-50 ml capacity.

$-\mathrm{NaOH}(4 \%)$ was added to the homogenate in a volume equal to that of specimens.
-The tubes were sealed and shaken on a shaker for 25 $\min$.

-Then sterile distilled water was added to the top ring in the centrifuge tube and centrifuged at $3000 \mathrm{rpm}$ for $20 \mathrm{~min}$.

-Then the supernatant was discarded and the sediment was re-suspended in $1 \mathrm{ml}$ of sterile normal saline.

-The prepared sediment was inoculated into two slants of Lowenstein-Jensen medium. Inoculated medium was incubated at $30^{\circ} \mathrm{C}$ for 8 weeks.

II. Recent method [MB-Redox System Heipha Diagnostica, Germany (Din, 1996)]:

-Before inoculation, the MB-Redox tubes were wormed up at least to room temperature $\left(25-30^{\circ} \mathrm{C}\right)$.

- $0.25 \mathrm{ml}$ of previously processed specimens was inoculated into each of the MB-Redox tubes under aseptic condition using an insulin syringe and the tubes were closed tightly before incubation in an incubator at $30^{\circ} \mathrm{C}$.

-The MB-Redox tubes were examined after 24 hours incubation, the first time, thereafter twice weekly at intervals of 3 to 4 days. After 4 weeks incubation, the culture was examined once weekly.

-Each examination generally was performed in two steps:

a. Examination of the culture medium and the sediment without tilting the tube.

b. Examination of the sediment particles after slight tilting of the tube.

-The appearance of pink-red- or violet-coloured particles was a strong indication of the presence of mycobacteria also a complete colorization of the culture medium indicated the presence of mycobacteria.

-The presence of mycobacteria was confirmed by examination of a Kinyoun's Z.N. stained smear microscopically.

\section{Identification of the recovered mycobacteria:}

Identification of suspected colonies was carried out depending upon: morphological characters (Cruickshank et al., 1973), rate of growth (Runyoun, 1959), pigmentation (Songer and Trautman, 1980), growth at different temperatures and biochemical tests (Kubica, 1973) for full identification of the isolated mycobacterium species namely Niacin test, nitrate reduction test, hydrolysis of Tween 80 , arylsulfatase test, iron uptake, tolerance to $5 \%$ sodium chloride, 
growth on MacConkey's agar, urease test and thiophen-2 carboxylic acid hydrazide growth inhibitor.

\section{Results}

\section{Results of mycobacterial isolation:}

All of the 240 Nile tilapia from Nile River in Sohage, Assuit and Qena Governorates were free from mycobacteria and no mycobacteria were detected by microscopic smear or by culture. Three isolates of atypical mycobacteria were recovered from water samples from the Nile. Four other isolates were isolated from aquarium water from different aquaria in shops present in these three Governorates. Also 11 isolates of atypical mycobacteria were isolated from fantail goldfish raised in aquaria (Table 1).

Table (1): Results of mycobacterial isolation from fish and water

\begin{tabular}{|c|c|c|c|c|c|c|}
\hline \multicolumn{2}{|l|}{ Samples } & \multicolumn{2}{|c|}{$\begin{array}{l}\text { Direct } \\
\text { smear* }\end{array}$} & \multicolumn{2}{|c|}{$\begin{array}{l}\text { Growth on } \\
\text { culture media }\end{array}$} & \multirow{2}{*}{ Isolates } \\
\hline Type & No. & No. & $\%$ & No. & $\%$ & \\
\hline Tilapia fish & 240 & 0 & 0 & 0 & 0 & $\begin{array}{l}\text { Negative } \\
\text { culture }\end{array}$ \\
\hline $\begin{array}{l}\text { Water samples } \\
\text { from Nile }\end{array}$ & 50 & 3 & 6 & 3 & 6 & $\begin{array}{l}\text { Isolates No. } \\
16,17 \text { and } 18\end{array}$ \\
\hline $\begin{array}{l}\text { Diseased gold } \\
\text { fish (Ascitis and } \\
\text { tissue from } \\
\text { ulcer) }\end{array}$ & 60 & $\begin{array}{l}6 \\
3\end{array}$ & $\begin{array}{c}10 \\
5\end{array}$ & $\begin{array}{l}7 \\
4\end{array}$ & $\begin{array}{c}11.66 \\
6.66\end{array}$ & $\begin{array}{c}\text { Isolates No. } \\
1,2,3,4,5,6 \\
\text { and } 10 \\
\text { Isolates No. } \\
7,8,9 \text { and } 15\end{array}$ \\
\hline Aquarium water & 40 & 4 & 10 & 4 & 10 & $\begin{array}{c}\text { Isolates No., } \\
11,12,13 \\
\text { and } 14\end{array}$ \\
\hline
\end{tabular}

* Acid fast smear

\section{Results of identification of the isolated acid-fast bacteria:}

From morphological, growth characteristics and biochemical characteristics of the isolated mycobacteria, the isolates No. 1, 2, 3, 9, 11 and 15 were identified as M. marinum, while isolates No. 10 , 12, 13 and 14 were identified as M. fortuitum (Photo 1, 2 and Table 2).

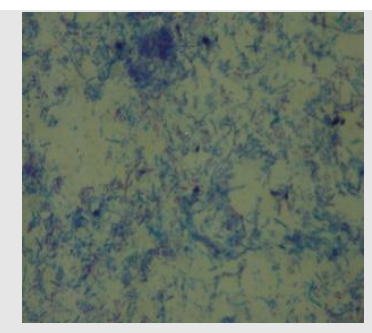

Photo. 1

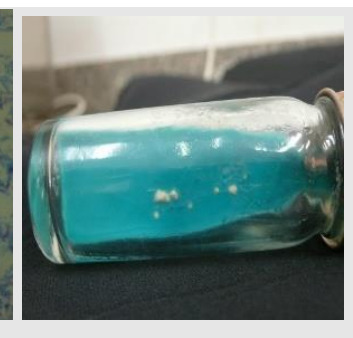

Photo. 2
Photo (1): A film from Nile water sediment showing acid fast bacilli. Photo (2): Isolated atypical mycobacteria ( $M$. fortuitum) on Lowenstein-Jensen medium (4 days post inoculation).

3. Results of comparison between using MB-redox system and Lowenstein-Jensen medium for recovery of mycobacteria:

The recovery rate of MB-Redox system for mycobacteria was $12 \%$, while in case of conventional method (L-J medium) was $10.66 \%$ (Table 3).

\section{Results of comparison of time for detection of different mycobacteria grew on MB-redox system and Lowenstein-Jensen medium:}

The mean time for detection of M. marinum was 3-7 days and 7-10 days for MB-Redox system and Lowenstein-Jensen medium, respectively, with the average of days for detection was 5 days for MBRedox and 8.5 days for L-J medium. The mean time for detection of M. fortuitum was 1-3 days and 3-6 days for MB-Redox system and L-J medium, respectively with an average of 2 days for MB-Redox and 4.5 days for L-J medium. While the mean time for detection of other unidentified atypical mycobacteria was 3-8 days and 3-14 for MB-Redox system and L-J medium, respectively with an average of 5.5 days for MB-Redox system and 8.5 days for L-J medium (Table 4).

\section{Results of detection of mycobacterial growth on MB-redox system at different times:}

As illustrated in Table (5), the color of MB-Redox tubes appeared yellow at time of inoculation changed to faint pink color after one day then pink colored medium after 3 days. Colonies appeared as red particles on the surface at the third day. After 15 days, the color of MB-Redox media changed to yellow with red particles on the surface (Photo.3 and Table 5). 
Table (2): Results of physical and biochemical identification of the isolated acid fast bacilli from diseased fish and water*

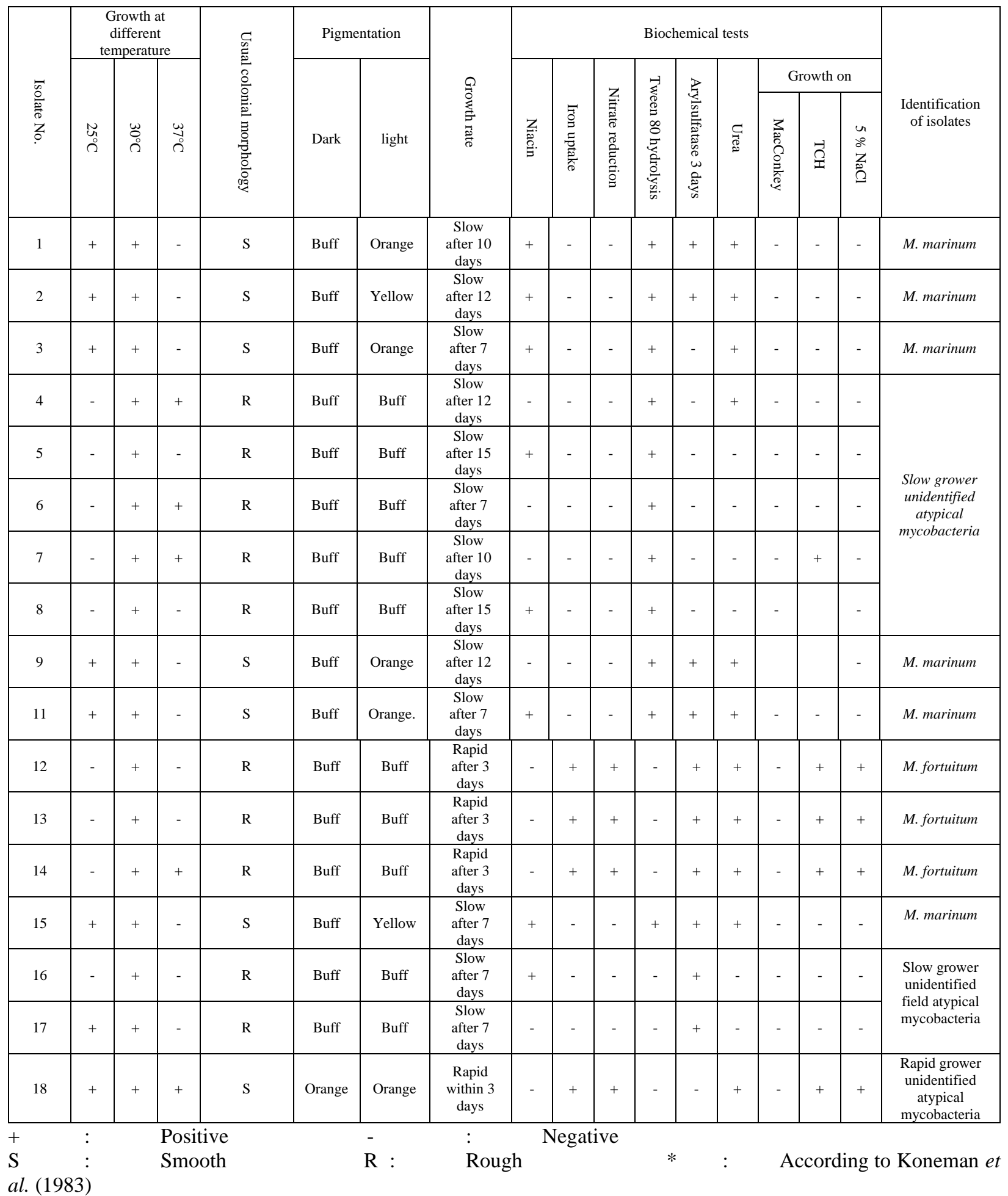


Table (3): Results of comparison between the recovery rate of mycobacteria by using MB-Redox system and conventional method from 150 processed specimens.

\begin{tabular}{|c|c|c|c|c|c|c|c|}
\hline \multicolumn{2}{|c|}{ Specimens } & \multicolumn{2}{|c|}{ AFB in direct smear } & \multicolumn{4}{|c|}{ Positive culture } \\
\hline \multirow[t]{2}{*}{ Types } & \multirow[t]{2}{*}{ No. } & \multirow[t]{2}{*}{ No. } & \multirow[t]{2}{*}{$\%$} & \multicolumn{2}{|c|}{ MB-Redox system } & \multicolumn{2}{|c|}{$\begin{array}{c}\text { Conventional culture medium } \\
(\mathrm{L}-\mathrm{J})\end{array}$} \\
\hline & & & & No. & $\%$ & No. & $\%$ \\
\hline Skin ulcer & 15 & 3 & 20 & 4 & 26.6 & 3 & 20 \\
\hline Ascitic fluids & 4 & 2 & 50 & 2 & 50 & 1 & 25 \\
\hline Liver and spleen & 41 & 4 & 9.7 & 5 & 12.1 & 5 & 12.1 \\
\hline Aquarium water & 40 & 4 & 10 & 4 & 10 & 4 & 10 \\
\hline Nile water & 50 & 3 & 6 & 3 & 6 & 3 & 5 \\
\hline Total & 150 & 16 & 10.66 & 18 & 12 & 16 & 10.66 \\
\hline
\end{tabular}

L-J: Lowenstein - Jensen medium. AFB: Acid fast bacilli

Table (4): Results of comparison of time for detection of different mycobacteria grew on MB-Redox system and Lowenstein - Jensen medium

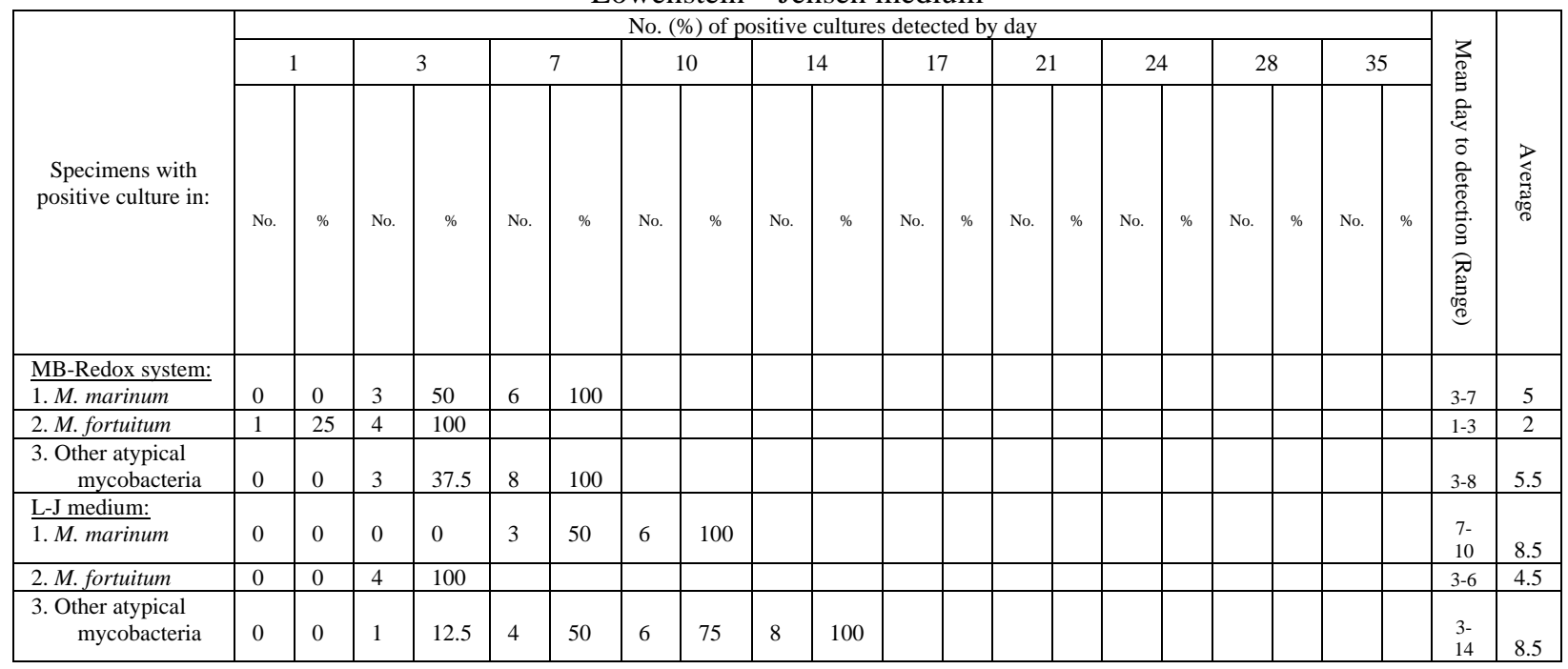

Table (5): Results of using MB-Redox system for detection of mycobacterial growth at different times.

\begin{tabular}{|c|c|c|c|c|c|}
\hline No. of specimens & $\begin{array}{c}\text { At time of } \\
\text { inoculation }\end{array}$ & After one day & 3 days & 7 days & 15 days \\
\hline $13 *$ & $\begin{array}{c}\text { Yellow colored } \\
\text { medium }\end{array}$ & $\begin{array}{c}\text { Faint pink colored } \\
\text { medium }\end{array}$ & $\begin{array}{c}\text { Pink colored } \\
\text { medium with very } \\
\text { delicate red } \\
\text { particles on the } \\
\text { surface }\end{array}$ & $\begin{array}{c}\text { Pink red colored } \\
\text { medium with } \\
\text { delicate red } \\
\text { particles on the } \\
\text { surface }\end{array}$ & $\begin{array}{c}\text { Yellow colored } \\
\text { medium with } \\
\text { delicate red } \\
\text { particles on the } \\
\text { surface }\end{array}$ \\
\hline $5 * *$ & $\begin{array}{c}\text { Yellow colored } \\
\text { medium }\end{array}$ & $\begin{array}{c}\text { Faint pink colored } \\
\text { medium }\end{array}$ & $\begin{array}{c}\text { Pink colored } \\
\text { turbidity of media } \\
\text { with fine red } \\
\text { particles on the } \\
\text { surface }\end{array}$ & $\begin{array}{c}\text { Pink colored media } \\
\text { with fine red } \\
\text { particles on the } \\
\text { surface }\end{array}$ & $\begin{array}{c}\text { Yellow colored } \\
\text { media with fine red } \\
\text { particles on the } \\
\text { surface }\end{array}$ \\
\hline
\end{tabular}

*: $\quad$ Identified as $M$. marinum $(n=6)$ and unidentified slow grower $(n=7)$ atypical mycobacteria **: $\quad$ Identified as $\boldsymbol{M}$. fortuitum $(\mathrm{n}=4)$ and unidentified rapid grower $(\mathrm{n}=1)$ atypical mycobacteria 


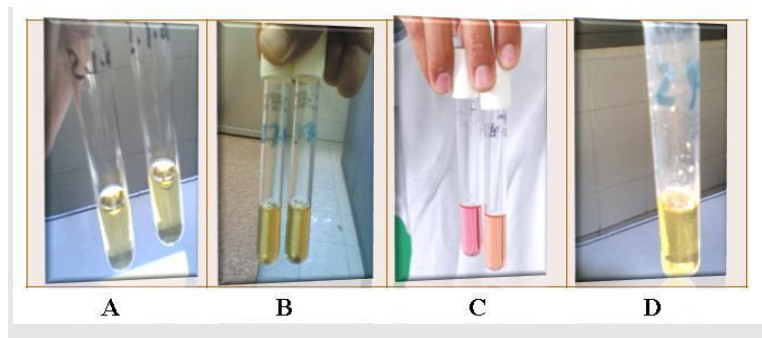

Photo (3): MB-Redox system inoculated with M. marinum. A: MB-Redox tube before inoculation and control, B: One day after inoculation, C: 7 days after inoculation, D: 15 days after inoculation.

\section{Discussion}

Piscine mycobacteriosis is a serious and often lethal disease of fish, affecting a wide range of species globally both in culture and wild settings, caused by several species of the genus Mycobacterium, the disease has been received considerable attention in recent years because of the discovery of new species in piscine hosts, epizootics in wild fisheries, and the ability of a few species to infect humans (Jacobs et al., 2009).

In the present work, eleven isolates of atypical mycobacteria were isolated from goldfish $(18.3 \%)$. Also four isolates were obtained from aquarium water $(10 \%)$ and three other isolates were obtained from Nile water (6\%), while all Nile tilapia from Nile River in Sohage, Assuit and Qena Governorates were free from mycobacteria.

On applying the morphological, cultural and biochemical studies of the isolated acid-fast bacilli obtained from aquarium fish, aquarium water and water from the Nile (Table 2, Photo. 1 and 2). Six isolates were identified as $M$. marinum and 4 isolates were identified as $M$. fortuitum. Decostere and Hermons (2003) reported that $M$. marinum, $M$. fortuitum and $M$. chelonae are the main etiological agents of fish mycobacteriosis. The former name is the closest genetic relative of the $M$. tuberculosis complex (Tobin and Ramakrishnan, 2008) and is a natural pathogen of ectotherms.

The results of comparison between using of the recent MB-Redox system and the conventional L-J medium for recovery of mycobacteria (Table 3) revealed that the recovery rate of $\mathrm{MB}-\mathrm{Redox}$ for mycobacteria (12\%) was higher than that of the conventional L-J medium $(10.66 \%)$. These results agree with the findings of Akos and Pal (1999), Emmanuelle et al. (1999), Piersimoni et al. (1999) and Samra et al. (2000) who reported that the recovery rate of AFB by MB-Redox system was higher than the recovery rate of AFB by L-J medium. MB-Redox system gave reliable results, offering the advantages of ready to use MB-Redox tubes in which the antibiotic supplement is already incorporated, easy and immediate reading of the results. Also, as this system does not contain any radioactive substance in comparison with BACTEC 460 TB system so results can be confirmed with acid fast staining and conventional and molecular tests.

The obtained results in Table (4) revealed that the mean time for detection of $M$. marinum, $M$. fortuitumand other unidentified atypical mycobacteria that grew on MB-Redox system was 3-7 days, 1-3 days and 3-8 days, respectively. The mean time for recovery of $M$. marinum, $M$. fortuitum and unidentified atypical mycobacteria that grew on L-J medium in the present work was 7-10 days, 3-6 days and 3-14 days, respectively. The mean times for detection of different Mycobacterium species in MBRedox system were shorter than that observed with LJ medium. These results agree with that reported by Wolf et al. (1998), Abe et al. (1999), Akos and Pal (1999) and Emmanuelle et al. (1999). Also, the present results agreed with the finding of Heifets et al. (2000) who recorded that the time for recovery of NTM in MB-Redox tube was 6.9 days for smear positive specimens and 15.5 days for smear negative specimen. They added that neither MB-Redox system nor other recent techniques (MGIT and BACTEC 460) should be used instead of a solid media (L-J medium) rather than they should be used in addition to it, the combined use of MB-Redox with L-J medium improved the overall level of recovery of mycobacteria from the specimens and provides rapid detection of mycobacterial growth for up to $88 \%$ of the culture positive specimens.

The results of detection of mycobacterial growth on MB-Redox tubes at different times (Table 5 and Photo. 3 ) revealed change in color of medium from yellow to faint pink after 24 hours and pink after 3 days, $M$. marinum grew as delicate red colored particles on the surfaces. $M$. fortuitum and some other mycobacteria grew as fine red particles. After 15 days the medium changed to yellow color. These findings are in agreement with Satio (1998) and Martin et al. (2006) who recorded that MB-Redox system is easily detectable by eye on the basis of their formazan (insoluble salt) color (pink-red-violet). The staining of 
the particles is stable for 2 weeks after which a decolorization may occur (yellow color).

In conclusion and from these aforementioned results it was found that atypical mycobacteria $(M$. fortuitum and $M$. marinum) are present in aquarium fish and water as well as in fresh water (Nile water). This is very important due to the mycobacterium infected fish or contaminated water can cause disease in human so that it is very important to avoid contact between the atypical mycobacteria of fish and other mycobacteria (M. tuberculosis complex) to avoid the possible transfer of drug resistance in between. Comparison between conventional method and recent method (MB-Redox system) was done to determine the ideal techniques for recovery of these agents.

\section{Conflict of interest}

The authors declare that they have no conflict of interest.

\section{References}

Abe, C.; Masako, W. and Kazue, H. (1999). Comparison of newly developed MB-Redox system with MGIT and 2\% Ogaw egg media for recovery of mycobacteria in clinical specimens. Kekkaku, 74(10): 707-713.

Akos, S. and Pal, M. (1999). Comparison of MGIT with MB-Redox, L-J medium and Middlebrook 7H11 for recovery of mycobacteria from clinical specimens. Am. Soc. Microbiol., 102: 426.

Cruickshank, R.; Duguid, J. P and Marmion, B. P. (1973). Staining Method, Chap. 2: p.331-357 and Test for Identification of Bacteria, Chap. 7: 170:189. Med. Microb. Pub. Churchil Liviengstone Edingurgh London and New York.

Decostere, A. and Hermons, K. (2003). Piscin Mycobacteriosis: a literature review covering the agent and the disease it causes in fish and human. J. Vet. Mic., 99(3-4):159-166.

Din (Dutch Industrial Normalization) (1996). Kulturelle Methodenzum Nachweias von Mykobacterien Medizinsche MikrobiologieTuberkulosediagnostika, pp. 3-12.

Emmanuelle, C.; Claudine, W. and Chantal, T.P. (1999). Evaluation of the new MB-Redox system for detection of mycobacteria. J. Clin. Microbiol., 37(6): 2013-2015.

Heifets, L.; Linder, T.; Sanzhez, T.; Spencer, D. and Brennan, J. (2000). Two liquid medium systems MGIT and MB-Redox tube for Mycobacterium tuberculosis isolation from sputum specimens. J. Clin. Microbiol., 38(3): 1227-1230.

Jacobs, J.M.; Stine, C.B.; Baya, A.M. and Kent, M.L. (2009). A review of mycobacteriosis in marine fish. J. Fish Dis., 32(2): 119-130.

Koneman, E.W.; Allen, S.D.; Dowell, V.R. and Sommers, H.M. (1983). Diagnostic Microbiology, second edition, J.B. Lippincott Company, Philadelphia, St. Lauis, London, San Paulo, New York, Sydney.

Kubica, G. B. (1973). Differential identification of mycobacterium. VII-key features for identification of clinically significant mycobateria. Am. Rev. Resp. Dis., 107: 9-12.

Macrì, D.; Lo Verde,V.; Mancuso, S.; Reale Passantino, A. and Marino, F. (2008). Mycobacteriosis in ornamental fish. Case reports in Sicily and medical-legal considerations. Vet. Res. Comm., 32(1): 215-217.

Marks, J. (1972): Ending the routine guinea pig test. Tuberculosis, 53: 31-34.

Martin, L.; Otera, F. and Maza, A. (2006). New diagnostic tools for tuberculosis. Int. J. TB and Lung Dis., 4(12): 182-188.

Piersimoni, C.; Scarparo, C.; Cichero, P.; DePezzo, M.; Covelli, I.; Gesu, G. and Nista, D. (1999). Multicenter evaluation of the MB-Redox medium compared with radiometric BACTEC system, mycobacteria growth indicator tube (MGIT) and Lowenstein-Jensen medium for detection and recovery of acid fast bacilli. Diagn. Microbiol. Infect. Dis., 34(4): 393-399.

Runyoun, E.H. (1959): Anonymous mycobacterium pulmonary disease. Med. Clin N.A.M., 43: 273-280.

Samra, Z.; Kaufman, L.; Bechor, J. and Bahar, J. (2000): Comparative study of three culture systems for optimal recovery of mycobacteria from different clinical specimens. Eur. J. Clin. Microbiol. Infect. Dis, 19(10): 750-754. 
Satio, H. (1998): Laboratory media for the cultivation of tubercle bacillus. Kekkaku, 73(5): 329-337.

Songer, D. G. and Trautman, R. J. (1980): A simple key for identification of the mycobacteria. Am. Ass. Vet. Lab. Dis. Ann. Proc., 23: 245-250.

Tobin, D.M. and Ramakrishnan, L. (2008): Comparative pathogens of Mycobacterium marinum and Mycobacterium tuberculosis. Cell Microbiol., 10(5):1027-1039.

Wolf, H.; Naumann, L.; Lehn, N. and Reischl, U. (1998). New rapid cultural non-radiometric methods for the detection of mycobacteria. Gen. Meet. Am. Soc. Microbiol., 4(8): 568-597.

\section{Cite this paper}

Mahmoud E. Hatem, Essam A. Nasr, Mohamed S. Marzouk, Fatma A. S. Mohamed, Mahmoud H. Mohmed. 2021. Comparison between conventional and recent culture methods for detection of fish mycobacteria. New Valley Veterinary Journal. 1(1):18. 